\title{
The Efficiency of Type-Specific High-Risk Human Papillomavirus Models in the Triage of Women with Atypical Squamous Cells of Undetermined Significance
}

This article was published in the following Dove Press journal:

Cancer Management and Research

\section{Yangzhen Wang' \\ Shanshan Gao' \\ Yuxia Wang' \\ Fuchun Chen ${ }^{2}$ \\ Hailong Deng ${ }^{3}$ \\ Yongfang Lu (1D)}

'Department of Central Laboratory, Longyan First Hospital Affiliated to Fujian Medical University, Longyan, People's

Republic of China; ${ }^{2}$ Department of Gynecology, Longyan First Hospital Affiliated to Fujian Medical University, Longyan, People's Republic of China;

${ }^{3}$ Department of Pathology, Longyan First Hospital Affiliated to Fujian Medical University, Longyan, People's Republic of China
Correspondence: Yongfang Lu Department of Central Laboratory, Longyan First Hospital Affiliated to Fujian Medical University, Longyan, People's

Republic of China

Tel +86-597-2205918

Email lyongfanglu@163.com
Purpose: To evaluate the performance of different high-risk human papillomavirus (HR-HPV) genotype models in triaging women with cytological diagnosis of atypical squamous cells of undetermined significance (ASCUS).

Patients and Methods: A total of 36,679 Chinese women who underwent cytology and HR-HPV genotyping assessments during cervical cancer screening were enrolled in this study. Women with cytology-proven ASCUS were referred for further screening by colposcopy and biopsy. The study endpoint was histological detection of cervical intraepithelial neoplasia grade 2 or worse (CIN2+) at any of the follow-up visits. The sensitivity, specificity, positive predictive values (PPVs), negative predictive values (NPVs), positive likelihood ratio (PLR) and negative likelihood ratio (NLR) of different HR-HPV genotype combination models were estimated.

Results: In all, 1675 (4.9\%) women were identified as having ASCUS, 1454 women underwent colposcopy and biopsy, and 6.0\% (87/1454) women were identified as having CIN2+ lesions. Among those with ASCUS who were identified as having CIN2+, the HRHPV infection rate was $97.7 \%$, and the prevalence rates of HPV-16, $-18,-31,-33,-35,-39$, $-45,-51,-52,-56,-58,-59,-66$ and -68 were $48.3 \%, 8.0 \%, 6.9 \%, 4.6 \%, 1.1 \%, 2.3 \%$, $3.4 \%, 3.4 \%, 26.4 \%, 1.1 \%, 17.2 \%, 2.3 \%, 0.0 \%$ and $0.0 \%$, respectively. Compared to other HR-HPV-type combination models, the HPV16/18/31/33/52/58 model achieved a higher sensitivity [93.1 (87.8-98.4)], specificity [73.0 (70.7-75.4)], PPV [18.0 (14.5-21.5)], NPV [99.4 (98.9-99.9)], PLR [3.7 (3.1-3.8)] and NLR [0.06 (0.03-0.18)] for the triage of ASCUS patients, but the colposcopy referral rate $(30.9 \%)$ was significantly lower than that of the recommended HR-HPV model (44.0\%).

Conclusion: This study confirms that the specific HR-HPV genotype HPV16/18/31/33/52/ 58 is an alternative strategy for ASCUS triage and can effectively reduce the high burden of colposcopy referrals in China.

Keywords: human papillomavirus, high-risk, genotyping, atypical squamous cells of undetermined significance, cervical intraepithelial neoplasia

\section{Introduction}

Cervical cancer is the second most common cancer among women worldwide; ${ }^{1}$ however, among all cancers, cervical cancer is the easiest to prevent by screening. ${ }^{2}$ Thus, it is particularly important to optimize screening programs to reduce the burden of cervical cancer. Currently, guidelines tend to recommend "three steps" 
for the diagnosis of cervical lesions: liquid cytology and high-risk human papillomavirus (HR-HPV), colposcopy (an observation method that can identify potential premalignant and malignant lesions) and histology. ${ }^{3}$ Women may need further follow-up and/or treatment based on the severity of the lesion when atypical transformation is detected by cytology. Women with cytology-proven severe lesions should be referred and further examined according to the guidelines, which involve colposcopy and biopsy obtained under colposcopy. ${ }^{4,5}$ However, the management strategy for women with atypical squamous cells of undetermined significance (ASCUS) remains controversial. ${ }^{6}$ ASCUS is not a specific diagnosis of progression or disease; rather, this indistinct diagnosis encompasses normal, low-grade squamous intraepithelial lesions (LSIL), highsquamous intraepithelial lesions (HSIL) and invasive cancer. $^{7}$ The consensus guidelines proposed in 2006 recommend three alternative treatments for women with ASCUS who have abnormal cell smears: repeat the cervical smear twice at 6-month intervals, repeat HR-HPV DNA tests and perform colposcopy. ${ }^{8}$ All of these options are safe and effective, depending on the situation and available resources.

Although most cases of ASCUS resolve with no apparent clinical disease, some (15-20\%) are confirmed by histology to progress to cervical epithelial neoplasia (CIN) during follow-up. ${ }^{9}$ Previous research has indicated that the risk of CIN3 or worse (CIN3+) among women with ASCUS is significantly higher than that among women without ASCUS. ${ }^{10}$ Therefore, an accurate category for these patients is needed to determine who needs subsequent management.

The etiologic role of persistent infection with HR-HPV, which may contribute to the development of cervical cancer and its precursor, is well documented. ${ }^{11,12}$ Over the past 10 years, the assessment of HR-HPV genotype has been adopted to guide treatment for women with ASCUS, and colposcopy is recommended for all HR-HPV-positive cases. $^{13}$ However, this management strategy may be overtreatment ${ }^{13}$ because of the different carcinogenicities of the different HPV genotypes, as the role of HPV in the evolution of CIN and invasive cancer varies by HPV genotype. HPV16, 18, 31, 33, 35, 39, 45, 51, 52, 56, 58, 59 and 68 are categorized as carcinogenic, and HPV66 is likely to be carcinogenic. ${ }^{14}$ Infection with HPV16 and HPV18 was found in $71 \%$ of cervical cancer cases. ${ }^{15}$ A previous study ${ }^{16}$ indicated that among HPV-infected cases with initial normal cytology, the incidence of CIN2 or worse $(\mathrm{CIN} 2+)$ at 13.4 years of follow-up was as follows: $28.5 \%$ with HPV16 alone; $15.4 \%$ with HPV18 alone; $19.1 \%$ with type $33,18.2 \%$ with type $35,16.7 \%$ with type $58,15.7 \%$ with type $31,8.6 \%$ with type 51 , $8.5 \%$ with type $45,4.7 \%$ with type $52,3.6 \%$ with type 39 , and $2.8 \%$ with type 56 . These data show the necessity of selecting appropriate HR-HPV genotypes for triaging ASCUS patients.

The ideal triage strategy for ASCUS patients is to maximize sensitivity and specificity, thereby reducing the rate of misdiagnosis and unnecessary colposcopy. Thus, our research was conducted to assess the performance of different HPV genotypes in triaging ASCUS patients in a Chinese population and offers new parameters to design the optimal strategy for ASCUS patient triage.

\section{Patients and Methods Study Design and Participants}

A cervical cancer screening group of 36,679 women was set up from August 2015 to December 2019 in Fujian Province. All participants underwent cervical cytology and HPV genotyping assessments. Women who met the following criteria were enrolled in the study: age 21 years or older; history of sexual activity; not currently pregnant; no history of hysterectomy, cervical surgery, or cervical cancer treatment; and provided signed informed consent. The Ethical Committees of the Longyan First Hospital Affiliated to Fujian Medical University granted approval for this study (Approval number: 2019-020). The study was conducted in accordance with the Declaration of Helsinki. All participants in this study provided written informed consent.

\section{Screening Protocols}

Prior to registration, a well-trained health care worker obtained informed consent and performed a confidential questionnaire-based interview with each included woman, evaluating her medical and surgical history of cervical lesions and cervical cancer, marital status, education level, smoking history, drinking history, and fertility history.

All qualified participants underwent gynecological examinations of the vulva, vagina and cervix. A doctor used a speculum to examine and collect cervical cells with plastic brushes. The cervical cells were collected in ThinPrep $^{\circledR}$ Pap Test PreservCyt ${ }^{\circledR}$ Solution (Hologic Inc., Madison, WI, USA) and transported to the laboratory 
immediately, where they were stored at $4^{\circ} \mathrm{C}$. The ThinPrep ${ }^{\circledR}$ Cytologic Test (TCT) and HR-HPV genotyping test were performed on ThinPrep cervical cells.

If the liquid-based cytology test results showed ASCUS, colposcopy was performed within 10 weeks by an experienced gynecologist. A completely visible cervical transition area indicated satisfactory colposcopy; colposcopy exams that showed normal findings did not require a biopsy. In contrast, for women whose colposcopy evaluations revealed abnormalities, cervical biopsy was performed directly on the visible lesions. If colposcopy was unsatisfactory (the cervical transition area was not completely visible), endocervical curettage (ECC) was subsequently conducted (Figure 1). Women who were confirmed to be CIN2+ by biopsy histopathology were treated with conization/hysterectomy according to the American Society for Colposcopy and Cervical Pathology (ASCCP) guidelines, ${ }^{8}$ and histopathological examination was performed after conization/hysterectomy. The degree of cervical lesions was determined by the histopathology after conization/ hysterectomy.

\section{HR-HPV Genotyping Assay}

We performed an HR-HPV genotyping assay with the HybriMax HPV GenoArray Test Kit (Chaozhou Hybribio Limited Corp., Chaozhou, China) according to the manufacturer's instructions. Previous research ${ }^{17,18}$ confirmed that the HybriMax HPV GenoArray Test Kit is an effective HR-HPV genotyping detection method, which has been approved by the China Food and Drug Administration (CFDA) for cervical cancer screening in China. The test is based on a DNA amplification, flow-through hybridization and gene-chip method, which can identify and distinguish 14 types of HR-HPV DNA in cervical exfoliated cells to detect each genotype. Specific primers and probes were designed for the HR-HPV L1 gene target sequence. Each experiment had positive and negative quality controls and blank controls. The reference gene b-globulin was measured simultaneously to determine false negatives due to insufficient sampling or manipulation errors.

\section{Cytology and Histology}

The liquid-based cytology and Bethesda System (TBS) was used for the cytology test, and the CIN classification system was used for histology. For all histological results, the worst result was defined as the final result for each woman. If no biopsy was performed or if the histological result was negative, the woman was regarded as CIN-negative. Cell pathologists and histopathologists of Longyan First Hospital Affiliated to Fujian Medical University prepared and read the films. All liquid-based cytology and histopathology specimens were blinded and independently evaluated by two experienced cytopathologists and histopathologists. If the diagnoses differed, the sample was reviewed again, and a consensus diagnosis was obtained. A training session on colposcopy and histopathological diagnosis was convened to standardize the treatment protocols before the beginning of the study.

\section{Statistical Analysis}

Analyses were carried out using SPSS version 24.0 (IBM Corporation, New York, USA). All women with ASCUS underwent colposcopy and/or ECC. The number of HRHPV-positive ASCUS patients divided by the total number of ASCUS patients was regarded as the referral rate. When calculating the infection rate for a given HR-HPV genotype, we included all women with single and multiple infections who were positive for the genotype. The mean and standard deviation of the categorical variables and the values and percentages of the categorical variables were calculated. We calculated the odds ratio (OR) of age and different HPV types to evaluate the risk of CIN2+ among women with ASCUS. The sensitivity, specificity, positive predictive value (PPV), negative predictive value (NPV), positive likelihood ratio (PLR) and negative likelihood ratio (NLR) of the HR-HPV genotyping model for CIN2 + cervical lesions were also calculated and compared with the gold standard of the pathological diagnosis. All of these results were used to assess the accuracy and effectiveness of HR-HPV genotyping in the triage of women with ASCUS. The significance level was set at a P-value $<0.05$.

\section{Results}

Of the 36,679 women recruited, 34,532 were eligible and included in the study, 1675 (4.9\%) had confirmed ASCUS by cytology, and 1454 (4.2\%) who underwent colposcopy and/or biopsy were enrolled in this study. The characteristics of the ASCUS patients are shown in Table 1. The mean age of the women at enrollment was $38.6 \pm 10.6$ years (range 21-75 years). Approximately 92.2\% had completed primary or higher education, $97.3 \%$ of those enrolled denied having a history of smoking, $80.9 \%$ denied having a history of drinking, $35.8 \%$ had more than 2 pregnancies, and $14.7 \%$ had more than 2 deliveries. (Table 1) 


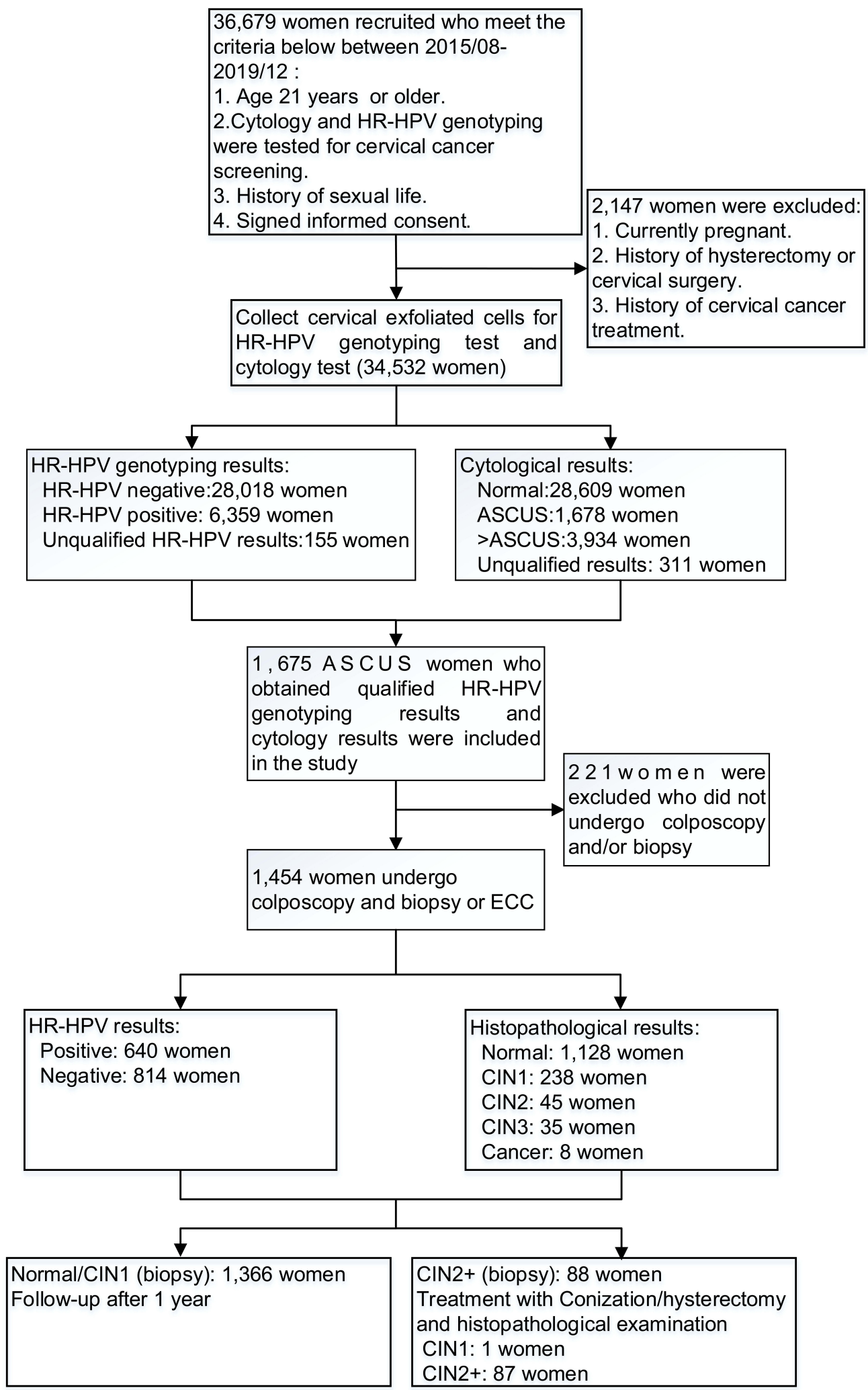

Figure I The flowchart of this study.

Abbreviations: HR-HPV, high-risk human papillomavirus, including types HPV-16, -18, $-31,-33,-35,-39,-45,-5 \mathrm{I},-52,-56,-58,-59,-66,-68$; ASCUS, atypical squamous cells of undetermined significance; CINI/2/3, cervical intraepithelial neoplasia grade I/2/3; ECC, endocervical curettage; CIN2+, cervical intraepithelial neoplasia grade 2 or worse. 
Table I Characteristics of the Study Population with CytologyProven ASCUS ( $\mathrm{N}=1454)$

\begin{tabular}{|c|c|c|}
\hline Characteristics & $\begin{array}{l}\text { No. of } \\
\text { Women } \\
(\mathrm{N}=1454)\end{array}$ & $\begin{array}{l}\text { Mean (x士s) or } \\
\text { Prevalence (\%) }\end{array}$ \\
\hline Age & 1454 & $38.6 \pm 10.6$ \\
\hline $21-30$ & 363 & $26.5 \pm 2.8(25.0 \%)$ \\
\hline $31-40$ & 508 & $35.3 \pm 3.0(34.9 \%)$ \\
\hline $4 I-50$ & 389 & $44.7 \pm 2.7(26.8 \%)$ \\
\hline $5 I-65$ & 171 & $56.2 \pm 4.3(11.8 \%)$ \\
\hline$>65$ & 23 & $70.4 \pm 3.5(1.6 \%)$ \\
\hline \multicolumn{3}{|l|}{ Level of education } \\
\hline Uneducated & 114 & 7.8 \\
\hline Primary education & 168 & 11.6 \\
\hline Middle school education & 768 & 52.8 \\
\hline$\geq$ University education & 404 & 27.8 \\
\hline \multicolumn{3}{|l|}{ Smoking } \\
\hline Yes - At least 3 times a week & 31 & 2.1 \\
\hline Yes - Less than 3 times a week & 8 & 0.6 \\
\hline No & 1415 & 97.3 \\
\hline \multicolumn{3}{|l|}{ Drinking } \\
\hline Yes - At least 2 times a week & 47 & 3.2 \\
\hline Yes - Less than 2 times a week & 231 & 15.9 \\
\hline No & 1176 & 80.9 \\
\hline \multicolumn{3}{|l|}{ Number of pregnancies } \\
\hline$\leq 2$ & 933 & 64.2 \\
\hline$>2$ & 521 & 35.8 \\
\hline \multicolumn{3}{|l|}{ Number of childbirths } \\
\hline$\leq 2$ & 1240 & 85.3 \\
\hline$>2$ & 214 & 14.7 \\
\hline \multicolumn{3}{|l|}{ HR-HPV infection } \\
\hline Positive & 640 & 44.0 \\
\hline Negative & 814 & 56.0 \\
\hline
\end{tabular}

Abbreviation: HR-HPV, high-risk human papillomavirus, including types HPV-16, $-|8,-3|,-33,-35,-39,-45,-5 \mid,-52,-56,-58,-59,-66,-68$.

The prevalence of HR-HPV among women with ASCUS was $44.0 \%(640 / 1454)$ (Table 1). Table 2 contains data on the prevalence rates of the different HPV genotypes among the women with ASCUS. HPV52 was the most prevalent, having been detected in 199 women (13.7\%), followed by HPV58 (99/1454, 6.8\%), HPV16 (95/1454, 6.5\%), HPV51 (79/1454, 5.4\%), HPV39 (49/ 1454, 3.4\%), and HPV18 (46/1454, 3.2\%).

The rate of HR-HPV infection increased with the severity of the pathological diagnosis. The prevalence of HR-HPV was $31.2 \%$ among ASCUS women with normal pathology and $100 \%$ among ASCUS women with CIN3 or cancer. Among the women with ASCUS and biopsy-
Table 2 Prevalence of Different HR-HPV Genotypes Among Women with ASCUS $(\mathrm{N}=1454)$

\begin{tabular}{|l|l|l|}
\hline $\begin{array}{l}\text { Triage Criteria } \\
\text { Using HR-HPV } \\
\text { Type Models }\end{array}$ & Positive n (\%) & Negative (n, \%) \\
\hline HR-HPV & $640(44.0 \%)$ & $814(56.0 \%)$ \\
HPVI6 & $95(6.5 \%)$ & $1359(93.5 \%)$ \\
HPVI8 & $46(3.2 \%)$ & $1408(96.8 \%)$ \\
HPV3I & $38(2.6 \%)$ & $1416(97.4 \%)$ \\
HPV33 & $36(2.5 \%)$ & $1418(97.5 \%)$ \\
HPV35 & $13(0.9 \%)$ & $1441(99.1 \%)$ \\
HPV39 & $49(3.4 \%)$ & $1405(96.6 \%)$ \\
HPV45 & $20(1.4 \%)$ & $1434(98.6 \%)$ \\
HPV5I & $79(5.4 \%)$ & $1375(94.6 \%)$ \\
HPV52 & $199(13.7 \%)$ & $1255(86.3 \%)$ \\
HPV56 & $45(3.1 \%)$ & $1409(96.9 \%)$ \\
HPV58 & $99(6.8 \%)$ & $1355(93.2 \%)$ \\
HPV59 & $40(2.8 \%)$ & $1414(97.2 \%)$ \\
HPV66 & $44(3.0 \%)$ & $1410(97.0 \%)$ \\
HPV68 & $45(3.1 \%)$ & $1409(96.9 \%)$ \\
\hline
\end{tabular}

Abbreviations: HR-HPV, high-risk human papillomavirus, including types HPV-16, $-18,-31,-33,-35,-39,-45,-51,-52,-56,-58,-59,-66,-68$. ASCUS, atypical squamous cells of undetermined significance.

confirmed CIN2, the prevalence rates of HR-HPV genotypes were as follows: HPV16, 38.6\%; HPV18, 6.8\%; HPV31, 13.6\%; HPV52, 36.4\%; HPV58, 20.5\%; and the 9 other types, $13.6 \%$. Among the women with histologically confirmed CIN3, the incidence rates of HR-HPV were 57.1\% (HPV16), 11.4\% (HPV18), 0\% (HPV31), 8.6\% (HPV33), 20.0\% (HPV52), 14.3\% (HPV58), and $14.4 \%$ (other 8 types) (Table 3 ).

Table 4 shows the distribution of different HR-HPV genotype models according to the histological diagnosis of CIN2+ in women with ASCUS. The prevalence rate of HR-HPV increased as more HPV genotypes were combined. The combination of HPV16/18/31/33/58/52 was able to identify $93.1 \%$ ( $\mathrm{p}<0.001)$ of women whose cytology was ASCUS but histologically proven CIN2+, similar to the HR-HPV model (97.7\%). However, based on HPV16/18 model, the rate was only $55.2 \%$.

We analyzed the factors that may predict CIN2+ in women with ASCUS, as shown in Table 5. HR-HPV-positive cases were associated with a 14.24 (95\% CI: 7.42-27.34, p<0.001) times greater probability of histological CIN2+ after adjustment for age. The risk was highest among women with HPV16 infection (OR: 37.38, 95\% CI: 20.58-67.88, p<0.001). HPV18 (OR: 4.62, 95\% CI: 1.63-13.11, p=0.004), HPV31 (OR: 3.39, 95\% CI: 1.07-10.70, p=0.038), HPV33 (OR: 2.22, 95\% CI: 1.46-8.11, p=0.043), HPV52 (OR: 4.49, 
Table 3 Distribution of HR-HPV Types According to Histological Diagnosis in Women with ASCUS (N=1454)

\begin{tabular}{|c|c|c|c|c|c|}
\hline \multirow{2}{*}{$\begin{array}{l}\text { HR-HPV Type } \\
\text { Models }\end{array}$} & \multicolumn{5}{|l|}{ Histological Diagnosis } \\
\hline & Normal $n=||$ 28, n (\%) & CINI n=239, n (\%) & CIN2 n=44, n (\%) & CIN3 n=35, n (\%) & Cancer $n=8, n(\%)$ \\
\hline HR-HPV & $352(3 \mid .2 \%)$ & $203(84.9 \%)$ & 42 (95.5\%) & $35(100.0 \%)$ & $8(100.0 \%)$ \\
\hline HPVI6 & $29(2.6 \%)$ & $24(10.0 \%)$ & $17(38.6 \%)$ & $20(57.1 \%)$ & $5(62.5 \%)$ \\
\hline HPVI8 & $21(1.9 \%)$ & 18 (7.5\%) & $3(6.8 \%)$ & $4(\mathrm{II} .4 \%)$ & $0(0.0 \%)$ \\
\hline HPV3I & $18(1.6 \%)$ & 14 (5.9\%) & $6(13.6 \%)$ & $0(0.0 \%)$ & $0(0.0 \%)$ \\
\hline HPV33 & $17(1.5 \%)$ & $15(6.3 \%)$ & $0(0.0 \%)$ & $3(8.6 \%)$ & I (I2.5\%) \\
\hline HPV35 & $9(0.8 \%)$ & $3(1.3 \%)$ & $0(0.0 \%)$ & I (2.9\%) & $0(0.0 \%)$ \\
\hline HPV39 & $32(2.8 \%)$ & $15(6.3 \%)$ & $2(4.5 \%)$ & $0(0.0 \%)$ & $0(0.0 \%)$ \\
\hline HPV45 & $9(0.8 \%)$ & 8 (3.3\%) & $2(4.5 \%)$ & $0(0.0 \%)$ & I (I2.5\%) \\
\hline HPV5I & $50(4.4 \%)$ & $26(10.9 \%)$ & I (2.3\%) & $2(5.7 \%)$ & $0(0.0 \%)$ \\
\hline HPV52 & $109(9.7 \%)$ & $67(28.0 \%)$ & $16(36.4 \%)$ & 7 (20.0\%) & $0(0.0 \%)$ \\
\hline HPV56 & $24(2.1 \%)$ & $20(8.4 \%)$ & $0(0.0 \%)$ & I (2.9\%) & $0(0.0 \%)$ \\
\hline HPV58 & 47 (4.2\%) & 37 (I5.5\%) & $9(20.5 \%)$ & 5 (14.3\%) & I (I2.5\%) \\
\hline HPV59 & $25(2.2 \%)$ & $13(5.4 \%)$ & I (2.3\%) & I (2.9\%) & $0(0.0 \%)$ \\
\hline HPV66 & $28(2.5 \%)$ & 16 (6.7\%) & $0(0.0 \%)$ & $0(0.0 \%)$ & $0(0.0 \%)$ \\
\hline HPV68 & $32(2.8 \%)$ & 13 (5.4\%) & $0(0.0 \%)$ & $0(0.0 \%)$ & $0(0.0 \%)$ \\
\hline
\end{tabular}

Abbreviations: ASCUS, atypical squamous cells of undetermined significance; HR-HPV, high-risk human papillomavirus, including types HPV-I6, $-18,-3 \mathrm{I},-33,-35,-39$, $-45,-51,-52,-56,-58,-59,-66,-68$; CIN, cervical intraepithelial neoplasia.

95\% CI: 2.42-8.31, p<0.001) and HPV58 (OR: 6.97, 95\% CI: 3.35-14.48, $\mathrm{p}<0.001)$ were also associated with a higher risk of histological CIN2+, whereas HPV35, HPV39, HPV45, HPV51, HPV56, HPV59, HPV66, and HPV68 did not have a significant effect on the risk. We also estimated the risk of CIN2+ according to different HPV genotype models among women with ASCUS (Table 5). The estimated ORs of CIN2+ were 8.89 (95\% CI: $1.21-65.15, \mathrm{p}=0.032)$ for the HPV16/18 model, 10.13 (95\% CI: 3.90-26.35, $\mathrm{p}<0.001)$ for the HPV16/ 18/58 model, 6.02 (95\% CI: 2.99-12.13, p<0.001) for the HPV16/18/52/58 model, $7.58 \quad(95 \% \quad$ CI: 3.88-14.79, $\mathrm{p}<0.001)$ for the HPV16/18/31/52/58/model, and $9.91(95 \%$ CI: $5.22-18.81, \mathrm{p}<0.001)$ for the HPV16/18/31/33/52/58 model.

The study endpoint was histological detection of cervical intraepithelial neoplasia grade 2 or worse $(\mathrm{CIN} 2+)$ at any of the follow-up visits. The sensitivity and NPVs of the HR-HPV genotype model increased, followed by those of the HPV16/18, HPV16/18/58, HPV16/18/52/58, HPV16/18/31/52/58, HPV16/18/31/33/52/58 and HRHPV models. However, as the number of HPV genotype combinations increased, the specificity, PPV, PLR and NLR decreased (Table 6). The sensitivity, specificity, PPV, NPV, PLR and NLR for detecting CIN2+ based on the HR-HPV model were 97.7\% (94.6-99.9), 59.4\% (56.8-62.0), 13.3\% (10.7-15.9), 99.8\% (99.4-100.0), 2.6 (2.2-2.6) and $0.04(0.01-0.15)$, respectively. The sensitivity, NPV and NLR of the HPV16/18/31/33/52/58 model (sensitivity: 93.1\% (87.8-98.4); NPV: 99.4\% (98.9-99.9); NLR: 0.06 (0.03-0.18)) were similar to those of the HRHPV model for identifying CIN2+ among women with ASCUS, but the specificity (73.0\% (70.7-75.4)) and PPV (73.0\% (70.7-75.4)) of the HPV16/18/31/33/52/58 model were higher than those of the HR-HPV model. In addition, the referral rate for the HPV16/18/31/33/52/58 model $(30.9 \%)$ was lower than that for the HR-HPV model (44.0\%) (Table 6).

\section{Discussion}

ASCUS is the most frequent abnormal finding by cytology during cervical screening and can indicate either an active benign lesion or potential malignancy. Histological results may vary widely. Thus, a method to determine the appropriate clinical management strategy for women with ASCUS is needed. In recent years, HR-HPV DNA testing has been included in cervical screening programs, which can both be used to triage ASCUS patients ${ }^{19,20}$ and be a part of screening combined with TCT. Different HRHPV genotypes are associated with different levels of risk for the progression of CIN to invasive cancer. The effectiveness of type-specific HR-HPV types in triaging ASCUS cases may vary by the specific combination of different HPV genotypes. Therefore, it is meaningful to identify the specific HR-HPV genotype model with higher sensitivity and specificity and lower referral rates to triage women with ASCUS. 
Table 4 Distribution of Different HR-HPV Genotype Models According to the Histological Diagnosis of CIN2+ in Women with ASCUS $(\mathrm{N}=1454)$

\begin{tabular}{|c|c|c|c|}
\hline \multirow{2}{*}{$\begin{array}{l}\text { HR-HPV Genotype } \\
\text { Models }\end{array}$} & \multicolumn{2}{|c|}{ Histological Diagnosis } & \multirow[t]{2}{*}{$P$-value } \\
\hline & $\begin{array}{l}\text { Normal/CIN I } \\
N=\mid 367, \text { n (\%) }\end{array}$ & $\begin{array}{l}\text { CIN2+ } \\
\text { N=87, } \\
\text { n (\%) }\end{array}$ & \\
\hline HR-HPV & $555(40.6 \%)$ & 85 (97.7\%) & $<0.001$ \\
\hline HPVI6 & $53(3.9 \%)$ & $42(48.3 \%)$ & $<0.001$ \\
\hline HPVI8 & 39 (2.9\%) & 7 (8.0\%) & 0.018 \\
\hline HPV3I & $32(2.3 \%)$ & $6(6.9 \%)$ & 0.025 \\
\hline HPV33 & 32 (2.3\%) & $4(4.6 \%)$ & 0.338 \\
\hline HPV35 & $12(0.9 \%)$ & $1(1.1 \%)$ & 0.794 \\
\hline HPV39 & 47 (3.4\%) & $2(2.3 \%)$ & 0.791 \\
\hline HPV45 & $17(1.2 \%)$ & $3(3.4 \%)$ & 0.216 \\
\hline HPV5I & $76(5.6 \%)$ & $3(3.4 \%)$ & 0.550 \\
\hline HPV52 & $176(12.9 \%)$ & $23(26.4 \%)$ & $<0.001$ \\
\hline HPV56 & $44(3.2 \%)$ & $\mathrm{I}(\mathrm{I} .1 \%)$ & 0.446 \\
\hline HPV58 & $84(6.1 \%)$ & $15(17.2 \%)$ & $<0.001$ \\
\hline HPV59 & $38(2.8 \%)$ & $2(2.3 \%)$ & 0.790 \\
\hline HPV66 & $44(3.2 \%)$ & $0(0.0 \%)$ & 0.169 \\
\hline HPV68 & $45(3.3 \%)$ & $0(0.0 \%)$ & 0.162 \\
\hline HPV $16 / 18^{a}$ & $90(6.6 \%)$ & $48(55.2 \%)$ & $<0.001$ \\
\hline HPVI6//8/58 & $167(12.2 \%)$ & $63(72.4 \%)$ & $<0.001$ \\
\hline HPVI6//8/52/58 & $326(23.8 \%)$ & 77 (88.5\%) & $<0.001$ \\
\hline HPVI6//8/3I/52/58 & $348(25.5 \%)$ & $80(92.0 \%)$ & $<0.001$ \\
\hline $\mathrm{HPVI} / 6 / 18 / 31 / 33 / 58 / 52^{\mathrm{e}}$ & $369(27.0 \%)$ & 81 (93.1\%) & $<0.001$ \\
\hline
\end{tabular}

Notes: ${ }^{2}$ Women with HPVI6 and/or HPVI8 infection. ${ }^{\mathrm{b}} \mathrm{All}$ women with positivity for HPVI6, HPVI8, or HPV58. 'All women with positivity for HPVI6, HPVI8, HPV52 or HPV58. ${ }^{\mathrm{d} A l l}$ women with positivity for HPVI6, HPVI8, HPV3I, HPV52 or HPV58. ${ }^{\mathrm{e} A l l}$ women with any positivity for HPVI6, HPVI8, HPV3I, HPV33, HPV52 or HPV58.

Abbreviations: $\mathrm{HR}-\mathrm{HPV}$, high-risk human papillomavirus, including types HPV-16, $-18,-3 \mid,-33,-35,-39,-45,-5 \mathrm{I},-52,-56,-58,-59,-66,-68$; CIN, cervical intraepithelial neoplasia; CIN2+, cervical intraepithelial neoplasia grade 2 or worse; ASCUS, atypical squamous cells of undetermined significance.

Previous studies of the relevance of HR-HPV in the triage of ASCUS patients have mainly focused on HPV16 and HPV18. Data on HR-HPV types other than HPV16 and HPV18 are rare and inconsistent. Lin et $\mathrm{al}^{21}$ evaluated the role of HPV16/18 and other HR-HPV types in ASCUS and LSIL triage. A study conducted by Jiang et $\mathrm{al}^{22}$ analyzed the performance of 10 types of HR-HPV in the triage of ASCUS patients aged 25 to 36 years. Our study discusses the triage efficiency of different HR-HPV genotype models (HR-HPV, HPV16/18, HPV16/18/58, HPV16/18/52/58, HPV16/18/31/ 52/58, HPV16/18/31/33/52/58) for ASCUS patients, from a Chinese population, whose biopsies proved CIN2+. We found that the HPV16/18/58/52/31/33 model has a relatively high sensitivity, specificity, PPV, and NPV but a lower referral rate than other HR-HPV genotype models.
Table 5 Factors Predicting CIN2+ in Women with ASCUS

\begin{tabular}{|c|c|c|c|c|}
\hline $\begin{array}{l}\text { HR-HPV } \\
\text { Types }\end{array}$ & $\begin{array}{l}\text { No. of } \\
\text { Women } \\
(\mathrm{N}=1454)\end{array}$ & OR $(95 \% \mathrm{Cl})$ & $\begin{array}{l}\text { OR }_{\text {adjust }} \\
(95 \% \mathrm{Cl})^{\mathrm{a}}\end{array}$ & P-value \\
\hline \multicolumn{5}{|l|}{ Age } \\
\hline $21-30$ & 363 & I (R) & I (R) & \\
\hline $31-40$ & 508 & $1.20(0.66-2.19)$ & $1.68(0.82-3.45)$ & 0.159 \\
\hline $41-50$ & 389 & $1.60(0.88-2.93)$ & $1.95(0.95-4.04)$ & 0.071 \\
\hline $51-65$ & 171 & $0.82(0.34-2.00)$ & $1.29(0.47-3.57)$ & 0.620 \\
\hline$>65$ & 23 & $1.83(0.40-8.40)$ & $2.11(0.22-19.79)$ & 0.514 \\
\hline \multicolumn{5}{|l|}{ HR-HPV } \\
\hline Negative & 814 & I (R) & I (R) & \\
\hline Positive & 640 & 62.18 & 14.24 & $<0.001$ \\
\hline & & (15.24-253.74) & $(7.42-27.34)$ & \\
\hline \multicolumn{5}{|l|}{ HPVI6 } \\
\hline Negative & 1359 & I (R) & I (R) & \\
\hline Positive & 95 & $23.14(14.00-38.24)$ & 37.38 & $<0.001$ \\
\hline & & & (20.58-67.88) & \\
\hline \multicolumn{5}{|l|}{ HPVI8 } \\
\hline Negative & 1408 & I (R) & I (R) & \\
\hline Positive & 46 & $2.98(1.29-6.87)$ & $4.62(1.63-13.11)$ & 0.004 \\
\hline \multicolumn{5}{|l|}{ HPV3I } \\
\hline Negative & 1416 & I (R) & I (R) & \\
\hline Positive & 38 & $3.09(1.26-7.60)$ & $3.39(1.07-10.70)$ & 0.038 \\
\hline \multicolumn{5}{|l|}{ HPV33 } \\
\hline Negative & 1418 & I (R) & I (R) & \\
\hline Positive & 36 & $2.01(0.70-5.82)$ & $2.22(1.46-8.11)$ & 0.043 \\
\hline \multicolumn{5}{|l|}{ HPV35 } \\
\hline Negative & 144| & I (R) & I (R) & \\
\hline Positive & 13 & $1.31(0.17-10.22)$ & $2.17(0.25-18.55)$ & 0.479 \\
\hline \multicolumn{5}{|l|}{ HPV39 } \\
\hline Negative & 1405 & I (R) & I (R) & \\
\hline Positive & 49 & $0.66(0.16-2.77)$ & $1.21(0.25-5.86)$ & 0.815 \\
\hline \multicolumn{5}{|l|}{ HPV45 } \\
\hline Negative & 1434 & I (R) & I (R) & \\
\hline Positive & 20 & $2.84(0.82-9.87)$ & $2.60(0.60-11.23)$ & 0.200 \\
\hline \multicolumn{5}{|l|}{ HPV5I } \\
\hline Negative & 1375 & I (R) & I (R) & \\
\hline Positive & 79 & $0.61(0.19-1.96)$ & $0.75(0.18-3.06)$ & 0.689 \\
\hline \multicolumn{5}{|l|}{ HPV52 } \\
\hline Negative & 1255 & I (R) & I (R) & \\
\hline Positive & 199 & $2.43(1.47-4.02)$ & $4.49(2.42-8.31)$ & $<0.001$ \\
\hline \multicolumn{5}{|l|}{ HPV56 } \\
\hline Negative & 1409 & I (R) & I (R) & \\
\hline Positive & 45 & $0.35(0.05-2.57)$ & $0.29(0.02-3.63)$ & 0.335 \\
\hline \multicolumn{5}{|l|}{ HPV58 } \\
\hline Negative & 2199 & I (R) & I (R) & \\
\hline Positive & 201 & $3.18(1.75-5.79)$ & $6.97(3.35-14.48)$ & $<0.001$ \\
\hline
\end{tabular}

(Continued) 
Table 5 (Continued).

\begin{tabular}{|c|c|c|c|c|}
\hline $\begin{array}{l}\text { HR-HPV } \\
\text { Types }\end{array}$ & $\begin{array}{l}\text { No. of } \\
\text { Women } \\
(\mathrm{N}=1454)\end{array}$ & OR $(95 \% \mathrm{Cl})$ & $\begin{array}{l}\text { OR }_{\text {adjust }} \\
(95 \% \mathrm{Cl})^{a}\end{array}$ & P-value \\
\hline $\begin{array}{l}\text { HPV59 } \\
\text { Negative } \\
\text { Positive }\end{array}$ & $\begin{array}{l}1414 \\
40\end{array}$ & $\begin{array}{l}\text { I (R) } \\
0.82(0.20-3.47)\end{array}$ & $\begin{array}{l}\text { I (R) } \\
0.90(0.19-4.32)\end{array}$ & 0.895 \\
\hline $\begin{array}{l}\text { HPV66 } \\
\text { Negative } \\
\text { Positive }\end{array}$ & $\begin{array}{l}1410 \\
44\end{array}$ & $\begin{array}{l}\text { I (R) } \\
1\end{array}$ & $\begin{array}{l}\text { I (R) } \\
\text { I }\end{array}$ & 1 \\
\hline $\begin{array}{l}\text { HPV68 } \\
\text { Negative } \\
\text { Positive }\end{array}$ & $\begin{array}{l}1409 \\
45\end{array}$ & $\begin{array}{l}\text { I (R) } \\
\text { I }\end{array}$ & $\begin{array}{l}\text { I (R) } \\
l\end{array}$ & 1 \\
\hline $\begin{array}{l}\text { HPVI6/18 } \\
\text { model }^{b} \\
\text { Negative } \\
\text { Positive }\end{array}$ & $\begin{array}{l}1316 \\
138\end{array}$ & $\begin{array}{l}\text { I (R) } \\
\text { I7.46 (10.88-28.04) }\end{array}$ & $\begin{array}{l}\text { I (R) } \\
8.89(1.21-65.15)\end{array}$ & 0.032 \\
\hline $\begin{array}{l}\text { HPVI6/18/ } \\
58 \text { model }^{c} \\
\text { Negative } \\
\text { Positive }\end{array}$ & $\begin{array}{l}1224 \\
230\end{array}$ & $\begin{array}{l}\text { I (R) } \\
\text { I8.86(II.47-31.01) }\end{array}$ & $\begin{array}{l}I(R) \\
10.13 \\
(3.90-26.35)\end{array}$ & $<0.001$ \\
\hline $\begin{array}{l}\text { HPVI6/18/ } \\
52 / 58 \\
\text { model }^{d} \\
\text { Negative } \\
\text { Positive }\end{array}$ & $\begin{array}{l}1051 \\
403\end{array}$ & $\begin{array}{l}\text { I (R) } \\
24.59(12.58-48.07)\end{array}$ & $\begin{array}{l}\text { I (R) } \\
6.02(2.99-12.13)\end{array}$ & $<0.001$ \\
\hline $\begin{array}{l}\text { HPVI6/18/ } \\
31 / 52 / 58 \\
\text { model }^{e} \\
\text { Negative } \\
\text { Positive }\end{array}$ & $\begin{array}{l}1026 \\
428\end{array}$ & $\begin{array}{l}\text { I (R) } \\
33.47(15.31-73.15)\end{array}$ & $\begin{array}{l}\text { I (R) } \\
7.58(3.88-14.79)\end{array}$ & $<0.001$ \\
\hline $\begin{array}{l}\text { HPVI6/18/ } \\
31 / 33 / 52 / 58 \\
\text { model }^{f} \\
\text { Negative } \\
\text { Positive }\end{array}$ & $\begin{array}{l}1004 \\
450\end{array}$ & $\begin{array}{l}\text { I (R) } \\
36.51(15.80-84.39)\end{array}$ & $\begin{array}{l}\text { I (R) } \\
9.91(5.22-18.81)\end{array}$ & $<0.001$ \\
\hline
\end{tabular}

Notes: ${ }^{a} O R$ values were adjusted for age, education level, smoking, drinking, number of pregnancies, and number of childbirths. ${ }^{b}$ Women with HPVI6 and/or HPVI8 infection directly referred for colposcopy and biopsy. ${ }^{\mathrm{C}} \mathrm{All}$ women with positivity for HPVI6, HPVI8, or HPV58 were directly referred for colposcopy and biopsy. ${ }^{\mathrm{d}}$ All women with positivity for HPVI6, HPVI8, HPV52 or HPV58 were directly referred for colposcopy and biopsy. ${ }^{\mathrm{e}} \mathrm{All}$ women with positivity for HPVI6, HPVI8, HPV3I, HPV52 or HPV58 were directly referred for colposcopy and biopsy. ${ }^{\mathrm{f}}$ All women with positivity for HPVI6, HPVI8, HPV3I, HPV33, HPV52 or HPV58 were directly referred for colposcopy and biopsy.

Abbreviations: OR, odds ratio; ASCUS, atypical squamous cells of undetermined significance; HR-HPV, high-risk human papillomavirus, including types HPV-16, -18 , $-3 \mathrm{I},-33,-35,-39,-45,-5 \mathrm{I},-52,-56,-58,-59,-66,-68$; $\mathrm{R}$, reference.

According to prior studies, the rate of ASCUS findings by cervical cytology ranges from $3 \%$ to $10 \% .{ }^{23-25} \mathrm{In}$ accordance with these results, our research showed that ASCUS accounted for $4.9 \%$ of all cytology results.
However, the prevalence of HR-HPV among women with ASCUS was quite inconsistent in different studies. Although one study reported that the prevalence rate of HR-HPV among women with ASCUS was $55.6 \%,{ }^{26}$ another study reported a prevalence rate of $18 \% .{ }^{27}$ In our study, we found that the prevalence rate of HR-HPV among ASCUS patients was 44\%. Moreover, among ASCUS patients whose biopsies proved CIN2+, the infection rates of HPV16, HPV18, HPV31, HPV33, HPV52 and HPV58 were $48.3 \%, 8.0 \%, 6.9 \%, 4.6 \%, 26.4 \%$ and $17.2 \%$, respectively. Our study also showed no HPV31 infection in ASCUS women with CIN3 and cervical cancer, which may be due to the small number of women we included. Previous studies showed that the risk of developing CIN3+ in HPV31-infected women was similar to that in HPV18-infected women, ${ }^{28}$ and even higher than that in HPV18-infected women. ${ }^{29}$ These findings suggest a higher possibility of progression to high-grade CIN in ASCUS patients who are infected with HPV16, HPV18, HPV31, HPV33, HPV52 or HPV58, which implies the significance of specific HPV genotype detection.

A meta-analysis ${ }^{2}$ compared the accuracy of the HRHPV test with repeated cytology for potential CIN2+ or CIN3+ in ASCUS patients. The results indicated that the HR-HPV test is more sensitive than repeated cytology in the triage of ASCUS patients, but there is no significant difference in specificity. Our study shows that the sensitivity of HR-HPV to detect CIN2+ among ASCUS patients is $97.7 \%$ but that the specificity is only $59.4 \%$. Therefore, triaging ASCUS patients with the recommended HR-HPV test will drive the evolution of referral rates and increase unnecessary costs and burden to patients. HPV16, HPV18, HPV31, HPV33, HPV52 and HPV58 are the most common genotypes in rural China, accounting for $88 \%$ of all infections, and infection with HPV16, HPV18, HPV31, HPV33, HPV52 and HPV58 among ASCUS patients is a higher risk factor for progressing to $\mathrm{CIN} 2+{ }^{30}$ This is consistent with our study results. Our data indicate that HPV16, HPV18, HPV31, HPV33, HPV52 and HPV58 are the most prevalent types among ASCUS patients with a histological diagnosis of CIN2+. Therefore, the risk of different HPV genotypes associated with CIN2+ among ASCUS patients was estimated.

We found that HR-HPV positivity was associated with a higher odds of CIN2+ histology. In the analysis of different HR-HPV genotypes, the risk of CIN2+ was highest among women with HPV16 infections. HPV18, HPV31, HPV33, HPV52 and HPV58 were also associated with the risk of 
Table 6 The Effect of Different HR-HPV Genotype Models in Triaging Women with ASCUS. (N=1454)

\begin{tabular}{|c|c|c|c|c|c|c|}
\hline Models & $\begin{array}{l}\text { HR-HPV } \\
\text { Model }^{\mathrm{a}}\end{array}$ & $\begin{array}{l}\text { HPVI6/18 } \\
\text { Model }^{\mathrm{b}}\end{array}$ & $\begin{array}{l}\text { HPVI6/18/58 } \\
\text { Model }^{c}\end{array}$ & $\begin{array}{l}\text { HPVI6/18/52/58 } \\
\text { Model }^{\text {d }}\end{array}$ & $\begin{array}{l}\text { HPVI6/I8/31/52/ } \\
58 \text { Model }^{\mathrm{e}}\end{array}$ & $\begin{array}{l}\text { HPVI6/I8/31/33/52/ } \\
58 \text { Model }^{f}\end{array}$ \\
\hline Sensitivity\% (95\% Cl) & 97.7 (94.6-99.9) & $55.2(44.7-65.6)$ & $72.4(63.0-81.8)$ & $88.5(81.8-95.3)$ & $92.0(86.2-97.7)$ & $93.1(87.8-98.4)$ \\
\hline Specificity\% (95\% Cl) & $59.4(56.8-62.0)$ & $93.4(92.1-94.7)$ & $87.8(86.0-89.5)$ & $76.2(73.9-78.4)$ & $74.5(72.2-76.9)$ & $73.0(70.7-75.4)$ \\
\hline PPV\% (95\% Cl) & $13.3(|0.7-| 5.9)$ & $34.8(26.8-42.7)$ & $27.4(21.6-33.2)$ & 19.1 (I5.3-22.9) & $18.7(15.0-22.4)$ & $18.0(\mid 4.5-21.5)$ \\
\hline NPV\% (95\% Cl) & $99.8(99.4-100.0)$ & $97.0(92.1-98.0)$ & $98.0(97.3-98.8)$ & $99.0(98.5-99.6)$ & $99.3(98.8-99.8)$ & 99.4 (98.9-99.9) \\
\hline PLR $(95 \% \mathrm{Cl})$ & $2.6(2.2-2.6)$ & $8.9(6.4-11.0)$ & $6.3(4.9-7.2)$ & $3.9(3.3-4.2)$ & $3.8(3.2-4.0)$ & $3.7(3.1-3.8)$ \\
\hline NLR $(95 \% \mathrm{Cl})$ & $0.04(0.01-0.15)$ & $0.48(0.38-0.61)$ & $0.31(0.22-0.44)$ & $0.15(0.08-0.27)$ & $0.11(0.05-0.22)$ & $0.06(0.03-0.18)$ \\
\hline Referral rate $\%$ (n/N) & $44.0(640 / / 454)$ & $9.5(138 / 1454)$ & $15.8(230 / / 454)$ & $27.7(403 / / 454)$ & $29.4(428 / / 454)$ & $30.9(450 / 1454)$ \\
\hline
\end{tabular}

Notes: aAll women with positivity for HPVI6, HPVI8, HPV 3I, HPV 33, HPV 35, HPV 39, HPV 45, HPV 5I, HPV 52, HPV 56, HPV 58, HPV 59, HPV 66 or HPV68 were directly referred for colposcopy and biopsy. ${ }^{b}$ Women with HPVI6 and/or HPVI8 infection directly referred to colposcopy and biopsy. ${ }^{\mathrm{C}} \mathrm{All}$ women with positivity for HPVI6, HPVI8, or HPV58 were directly referred for colposcopy and biopsy. ${ }^{\mathrm{d} A l l}$ women with positivity for HPVI6, HPVI8, HPV52 or HPV58 were directly referred for colposcopy and biopsy. ${ }^{\mathrm{e}} \mathrm{All}$ women with positivity for HPVI6, HPVI8, HPV3I, HPV52 or HPV58 were directly referred for colposcopy and biopsy. ${ }^{\mathrm{f}} \mathrm{All}$ women with any positivity for HPVI6, HPVI8, HPV3I, HPV33, HPV52 or HPV58 were directly referred for colposcopy and biopsy. ${ }^{\text {T}}$ The rate of referral for colposcopy among ASCUS women.

Abbreviations: HR-HPV, high-risk human papillomavirus, including types HPV-16, -18, $-31,-33,-35,-39,-45,-51,-52,-56,-58,-59,-66,-68$; PPV, positive predictive value; NPV, negative predictive value; PLR, positive likelihood ratio; NLR, negative likelihood ratio.

histologically proven CIN2+, whereas HPV35, HPV39, HPV45, HPV51, HPV56, HPV59, HPV66, and HPV68 did not have a significant effect on the risk. An increased risk for CIN2+ was observed when the model combined more genotypes. The estimated OR of HPV16/18/31/33/ 52/58 was 9.91 according to our research.

We also calculated the sensitivity, specificity, PPV, and NPV of each HPV genotype combination, including HPV16/ 18, HPV16/18/58, HPV16/18/52/58, HPV16/18/31/52/58, HPV16/18/31/33/52/58 and HR-HPV, to identify potential CIN2+ populations among ASCUS patients. Similar to previous results, ${ }^{31}$ HPV16/18 was much more specific than the HPV16/18/31/33/52/58 combination (93.4\% versus $73.0 \%$ ) but lost sensitivity (55.2\% versus $93.1 \%$ ), which implies a higher possibility of misdiagnosis. However, we found that the sensitivity of HPV16/18/31/33/52/58 to identify CIN2+ in ASCUS cases was higher than that of HPV16/18/ 31/52/58, HPV16/18/52/58, or HPV16/18/58 (93.1\% versus $92.0 \%, 93.1 \%$ versus $88.5 \%, 93.1 \%$ versus 72.4 , respectively) and that the specificity of HPV16/18/31/33/52/58 was higher than that of HR-HPV (73.0\% versus 59.4\%). Moreover, according to the current guidelines for cervical cancer screening, HR-HPV-positive ASCUS patients are referred for colposcopy, and the referral rate of HPV16/18/ $31 / 33 / 52 / 58$ was significantly lower than recommended for HR-HPV (30.9\% versus 44.0\%), which can effectively reduce the high burden of colposcopy referrals. Therefore, it is reasonable to believe that the HPV16/18/31/33/52/58 genotype model is an alternative triage strategy to identify CIN2+ among ASCUS patients in China.

The potential limitation of our study is that there may be overtreatment because we performed colposcopy and biopsy in all eligible ASCUS women included in the study. Although the purpose is to clarify their cervical lesions, most ASCUS women have not progressed to CIN2+ lesions. A second potential limitation is that our research was conducted in a single region, and we did not perform verification in other regions; thus, the results may not be generalizable to other places.

\section{Conclusion}

This study evaluated the effectiveness of type-specific HRHPV models in the triage of ASCUS patients in the large-scale population to date. Due to its high sensitivity, specificity, and minimal number of HR-HPV genotypes, we suggest the HPV16/18/31/33/52/58 genotype model as an alternative strategy for the triage of ASCUS patients. Developing specific HPV genotyping assays could significantly increase the costeffectiveness of screening. To validate our results, further work is needed to evaluate our novel HPV genotype model in external populations.

\section{Abbreviations}

ASCUS, atypical squamous cells of undetermined significance; HR-HPV, high-risk human papillomavirus; CIN, cervical intraepithelial neoplasia; $\mathrm{CIN} 2+$, cervical intraepithelial neoplasia grade 2 or worse; OR, odds ratio; PPV, positive predictive value; NPV, negative predictive value; TCT, ThinPrep ${ }^{\circledR}$ Cytologic Test.

\section{Acknowledgment}

The authors would like to thank all the investigators for their contributions to this trial. Above all, we are grateful to all patients who participated in this study. 


\section{Funding}

This work was supported by grants from the Fujian Provincial Natural Science Foundation of China (grant no. 2017J01254).

\section{Disclosure}

The authors declare no potential conflicts of interest.

\section{References}

1. Ferlay J, Soerjomataram I, Dikshit R, et al. Cancer incidence and mortality worldwide: sources, methods and major patterns in GLOBOCAN 2012. Int $J$ Cancer. 2015;136:E359-E86. doi:10.1002/ijc. 29210

2. Arbyn M, Roelens J, Simoens C, et al. Human papillomavirus testing versus repeat cytology for triage of minor cytological cervical lesions. Cochrane Database Syst Rev. 2013;3:CD008054.

3. Boardman LA, Goldman DL, Cooper AS, Heber WW, Weitzen S. $\mathrm{CIN}$ in pregnancy: antepartum and postpartum cytology and histology. J Reprod Med. 2005;50:13-18.

4. Wright TC, Cox JT, Massad LS, Twiggs LB, Wilkinson EJ. ASCCP Sponsored Consensus Conference. 2001 Consensus guidelines for the management of women with cervical cytological abnormalities. JAMA. 2002;287:2120-2129. doi:10.1001/jama.287.16.2120

5. Koh WJ, Abu-Rustum NR, Bean S, et al. Cervical Cancer, Version 3.2019, NCCN Clinical Practice Guidelines in Oncology. $J$ Natl Compr Canc Netw. 2019;17:64-84. doi:10.6004/jncen.2019.0001

6. Cox JT. Management of women with cervical cytology interpreted as ASC-US or as ASC-H. Clin Obstet Gynecol. 2005;48:160-177. doi:10.1097/01.grf.0000151571.91814.f3

7. Solomon D, Davey D, Kurman R, et al. The2001 Bethesda System: terminology for reporting results of cervical cytology. JAMA. 2002;287:2114-2119. doi:10.1001/jama.287.16.2114

8. Wright TC, Massad LS, Dunton CJ, et al. 2006 consensus guidelines for the management of women with abnormal cervical cancer screening tests. Am J Obstet Gynecol. 2007;197:346-355. doi:10.1016/j. ajog.2007.07.047

9. Kyrgiou M, Kalliala IE, Mitra A, et al. Immediate referral to colposcopy versus cytological surveillance for minor cervical cytological abnormalities in the absence of HPV test. Cochrane Database Syst Rev. 2017;1:CD009836.

10. Katki HA, Schiffman M, Castle PE, et al. Benchmarking CIN3+ risk as the basis for incorporating HPV and Pap cotesting into cervical screening and management guidelines. $J$ Low Genit Tract Dis. 2013;17:S28-S35. doi:10.1097/LGT.0b013e318285423c

11. Schlecht NF, Kulaga S, Robitaille J, et al. Persistent human papillomavirus infection as a predictor of cervical intraepithelial neoplasia. JAMA. 2001;286:3106-3114. doi:10.1001/jama.286.24.3106

12. Nobbenhuis MA, Walboomers JM, Helmerhorst TJ, et al. Relation of human papillomavirus status to cervical lesions and consequences for cervical-cancer screening: a prospective study. Lancet. 1999;354:20-25. doi:10.1016/S0140-6736(98)12490-X

13. Meijer CJLM, Snijders PJF. Human papillomavirus triage of women with atypical squamous cells of undetermined significance-reduction of overtreatment needed. JAMA Oncol. 2017;3:1310-1311. doi:10.1001/jamaoncol.2017.1522

14. Bouvard V, Baan R, Straif K, et al. A review of human carcinogensPart B: biological agents. Lancet Oncol. 2009;10:321-322. doi:10.1016/S1470-2045(09)70096-8

15. de Sanjose S, Quint WG, Alemany L, et al. Human papillomavirus genotype attribution in invasive cervical cancer: a retrospective cross-sectional worldwide study. Lancet Oncol. 2010;11:1048-1056. doi:10.1016/S1470-2045(10)70230-8
16. Kjær SK, Frederiksen K, Munk C, Iftner T. Long-term absolute risk of cervical intraepithelial neoplasia grade 3 or worse following human papillomavirus infection: role of persistence. $J$ Natl Cancer Inst. 2010;102:1478-1488. doi:10.1093/jnci/djq356

17. Tao P, Zheng W, Wang Y, Bian ML. Sensitive HPV genotyping based on the flow-through hybridization and gene chip. $J$ Biomed Biotechnol. 2012;2012:938780. doi:10.1155/2012/938780

18. Chen Q, Xie LX, Qing ZR, et al. Epidemiologic characterization of human papillomavirus infection in rural Chaozhou, eastern Guangdong Province of China. PLoS One. 2012;7:e32149. doi:10.1371/journal.pone.0032149

19. Jordan J, Arbyn M, Martin-Hirsch P, et al. European guidelines for quality assurance in cervical cancer screening: recommendations for clinical management of abnormal cervical cytology, part 1. Cytopathology. 2008;19:342-354. doi:10.1111/j.1365-2303.2008.00623.x

20. ACOG Committee on Practice Bulletins-Gynecology. ACOG practice bulletin no. 109: cervical cytology screening. Obstet Gynecol. 2009;114:1409-1420. doi:10.1097/AOG.0b013e3181c6f8a4

21. Lin CQ, Cui JF, Zhang X, Pan QJ, Chen W, Qiao YL. Human papillomavirus genotyping to predict the risk of cervical precancerous lesions or cancer in women with minor abnormal cytology in China. Acta Cytol. 2015;59:405-411. doi:10.1159/000441290

22. Jiang L, Zeng Y, Li J, et al. Performance of high-risk human papillomavirus testing in the triage of abnormal cervical cytology among Chinese younger women in Shanghai, China. Asian Pac J Cancer Prev. 2011;12:2963-2967.

23. Kurman RJ, Henson DE, Herbst AL, Noller KL, Schiffman MH. Interim guidelines for management of abnormal cervical cytology. The1992 national cancer institute workshop. JAMA. 1994;271:1866-1869. doi:10.1001/jama.1994.03510470070037

24. Piccoli R, Mandato VD, Lavitola G, et al. Atypical squamous cells and low squamous intraepithelial lesions in postmenopausal women: implications for management. Eur J Obstet Gynecol Reprod Biol. 2008;140:269-274. doi:10.1016/j.ejogrb.2008.05.007

25. Goksedef BP, Akbayir O, Baran SY, et al. Atypical squamous cells of undetermined significance in postmenopausal women: a comparative retrospective analysis. Eur $J$ Obstet Gynecol Reprod Biol. 2011;159:418-421. doi:10.1016/j.ejogrb.2011.07.027

26. Gage JC, Schiffman M, Solomon D, et al. Risk of precancer determined by HPV genotype combinations in women with minor cytologic abnormalities. Cancer Epidemiol Biomarkers Prev. 2013;22:1095-1101. doi:10.1158/1055-9965.EPI-12-1455

27. Fakhreldin M, Elmasry K. Improving the performance of reflex Human Papilloma Virus (HPV) testing in triaging women with atypical squamous cells of undetermined significance (ASCUS): a restrospective study in a tertiary hospital in United Arab Emirates (UAE). Vaccine. 2016;34:823-830. doi:10.1016/j.vaccine.2015. 12.011

28. Sand FL, Munk C, Frederiksen K, et al. Risk of CIN3 or worse with persistence of 13 individual oncogenic HPV types. Int $J$ Cancer. 2019;144:1975-1982. doi:10.1002/ijc.31883

29. Bonde J, Bottari F, Parvu V, et al. Bayesian analysis of baseline risk of CIN2 and $\geq \mathrm{CIN} 3$ by HPV genotype in a European referral cohort. Int J Cancer. 2019;145:1033-1041. doi:10.1002/ijc.32291

30. Wright TC Jr, Stoler MH, Parvu V, Yanson K, Cooper C, Andrews J. Risk detection for high-grade cervical disease using Onclarity HPV extended genotyping in women, $\geq 21$ years of age, with ASC-US or LSIL cytology. Gynecol Oncol. 2019;154:360-367. doi:10.1016/j. ygyno.2019.05.012

31. Tay TKY, Lim KL, Hilmy MH, et al. Comparison of the sensitivity and specificity of p16/Ki-67 dual staining and HPV DNA testing of abnormal cervical cytology in the detection of histology proven cervical intraepithelial neoplasia grade 2 and above (CIN2+). Malays J Pathol. 2017;39:257-265. 


\section{Publish your work in this journal}

Cancer Management and Research is an international, peer-reviewed open access journal focusing on cancer research and the optimal use of preventative and integrated treatment interventions to achieve improved outcomes, enhanced survival and quality of life for the cancer patient.

The manuscript management system is completely online and includes a very quick and fair peer-review system, which is all easy to use. Visit http://www.dovepress.com/testimonials.php to read real quotes from published authors.

Submit your manuscript here: https://www.dovepress.com/cancer-management-and-research-journal 